Georgia State University

ScholarWorks @ Georgia State University

$9-1-2012$

\title{
Is There a Plausible Theory for Decision under Risk? A Dual Calibration Critique
}

James Cox

Georgia State University

Vjollca Sadiraj

Georgia State University

Bodo Vogt

Franklin and Marshall College

Utteeyo Dasgupta

Follow this and additional works at: https://scholarworks.gsu.edu/excen_workingpapers

\section{Recommended Citation}

Cox, James; Sadiraj, Vjollca; Vogt, Bodo; and Dasgupta, Utteeyo, "Is There a Plausible Theory for Decision under Risk? A Dual Calibration Critique" (2012). ExCEN Working Papers. 57.

https://scholarworks.gsu.edu/excen_workingpapers/57

This Article is brought to you for free and open access by the Experimental Economics Center at ScholarWorks @ Georgia State University. It has been accepted for inclusion in ExCEN Working Papers by an authorized administrator of ScholarWorks @ Georgia State University. For more information, please contact scholarworks@gsu.edu. 


\title{
Is There a Plausible Theory for Decision under Risk? A Dual Calibration Critique
}

\author{
James C. Cox, Vjollca Sadiraj, Bodo Vogt, and Utteeyo Dasgupta
}

Forthcoming in Economic Theory 


\title{
Is There a Plausible Theory for Decision under Risk? A Dual Calibration Critique
}

\author{
James C. Cox
}

Economics Department and Experimental Economics Center, Georgia State University

14 Marietta St. NW, Atlanta, GA 30303, USA

Vjollca Sadiraj

Economics Department and Experimental Economics Center, Georgia State University

14 Marietta St. NW, Atlanta, GA 30303, USA

404-413-0193

404-413-0205

vsadiraj@gsu.edu

Bodo Vogt

Faculty of Economics and Management, Otto-von-Guericke University Magdeburg

Gebaude 22, Universitatplatz 2, 39106 Magdeburg, G22A-344, Germany

Utteeyo Dasgupta

Franklin and Marshall College

415 Harrisburg Pike, Lancaster, PA 17603, USA

Acknowledgements Financial support was provided by the National Science Foundation (grant numbers IIS0630805 and SES-0849590). Helpful comments and suggestions were provided by an anonymous referee.

\begin{abstract}
Can any prominent theory of decision under risk rationalize both small-stakes risk aversion and largestakes risk aversion? Do some prominent theories fail to rationalize patterns of same-stakes risk aversion? How do reference payoffs enter in the answer to these questions? What would be the characteristics of a theory of decision under risk that would be immune to calibration critique? We offer a theoretical duality analysis that addresses these questions. We report dual propositions and corollaries that calibrate the implications of nonlinear transformation of probabilities or payoffs (or both). We also report several experiments that provide data on the empirical relevance of the two types of calibration patterns.
\end{abstract}

Keywords risk aversion, calibration, duality, reference dependence, experiments

JEL Classification Numbers C91, D81 


\section{Introduction}

Models of risk averse decision-makers are applied in a wide variety of contexts. For example, expected utility models have recently been applied in research on principle-agent theory (Chade and de Serio 2002), risk aversion and prudence (Lajeri and Nielsen 2000; Eichner and Wagner 2003), bounds on utility (LiCalzi 2000; Zambrano 2008), non-location scale distributions (Boyle and Conniffe 2008), and frequentist perspective ( $\mathrm{Hu}$ 2009). Rank dependent models have recently been applied to research on savings decisions (Bleichrodt and Eeckhoudt 2005), monotone risk aversion (Chateauneuf, Cohen and Meilijson 2005), and medical insurance (Ryan and Vaithianathan 2003). Dual theory of expected utility and cumulative prospect theory have, respectively, recently been applied in research on incomplete preferences (Maccheroni 2004) and the St. Petersburg paradox (Rieger and Wang 2006). Our paper reports research on internal coherence problems that can arise in applications of such models.

Models of decision under risk represent risk preferences with utility functionals that are nonlinear in payoffs or nonlinear in probabilities or nonlinear in both. For example, expected utility theory represents risk aversion with concave utility of payoffs. The dual theory of expected utility (Yaari 1987) represents risk aversion with convex transformation of decumulative probabilities. Rank dependent utility theory (Quiggin 1993) and cumulative prospect theory (Tversky and Kahneman 1992) represent risk attitudes with nonlinear transformations of both payoffs and probabilities. The nonlinear transformations suggest questions about the internal coherence of the theoretical models.

Rabin (2000) demonstrated how modeling risk aversion with concave utility of payoffs can fail to provide a coherent theory of both small-stakes risk aversion and large-stakes risk aversion. ${ }^{1}$ Although Rabin's statement of the critique applies only to the expected utility of terminal wealth model, subsequent authors extended this payoffs calibration critique to a class of theories of decision under risk that have utility functionals that are nonlinear in payoffs. ${ }^{2}$ But the payoffs calibration arguments have no implications for nonlinear transformations of probabilities, which is an alternative way to model risk aversion. Sadiraj (2012) presents a probabilities calibration that demonstrates the implausible implications of nonlinear

\footnotetext{
${ }^{1}$ See Hansson (1988) for an earlier critique of expected utility theory with similar arguments.

${ }^{2}$ Several studies report payoffs calibration patterns that apply to models defined on (a) terminal wealth or (b) income. Studies that focus on terminal wealth models include Hansson (1988), Rabin (2000), Neilson (2001), and Safra and Segal (2008, 2009). Varying-payoffs calibrations for models defined on income are reported by Barberis, Huang, and Thaler (2006), Cox and Sadiraj (2006), and Rubinstein (2006).
} 
transformation of probabilities. Each of the distinct types of calibration (of nonlinear payoffs transformation or nonlinear probabilities transformation) by itself has implications of implausible risk aversion for theories of decision under risk that transform both payoffs and probabilities.

Together, the payoffs calibrations and the probabilities calibrations provide an answer to a central question raised by the calibration literature: What would be the characteristics of a theory of risk-avoiding behavior that is immune to both payoffs calibration and probabilities calibration arguments? This paper offers insights into answering this question with an analysis of decision theories based on duality. We show that the payoffs calibration patterns conform to the linearity in payoffs property of the utility functional for the dual theory of expected utility (Yaari 1987). In contrast, the probabilities calibration patterns conform to the linearity in probabilities property of the utility functional for expected utility theory (von Neumann and Morgenstern 1947). Our dual analysis reveals that Sadiraj's (2012) probabilities calibration patterns, together with Rabin's (2000) payoffs calibration patterns, provide an answer to the central question about the properties that would characterize a theory of risk-avoiding preferences that would not be called into question by calibration critique. A theory of risk preferences with functional that is linear in probabilities would be immune to the probabilities calibration critique. A theory of risk preferences with functional that is linear in payoffs or assumes variable reference payoffs would be immune to the payoffs calibration critique. Hence a theoretical model characterized by linearity in probabilities and variable reference payoffs would be immune to both of the dual calibration critiques. A version of the vintage model in Markowitz (1952) has the requisite properties to survive the dual critiques unscathed. In contrast, currently popular models are vulnerable to one or both types of calibration critique.

The fundamentality of the calibration literature ultimately rests on empirical validity of the patterns of risk aversion supposed in the two types of calibration propositions. To date, however, there has been argument about the "reasonableness" of calibration suppositions but no data from real-payoff, controlled experiments to inform the issue. This paper reports several experiments conducted in three countries (India, Germany, and the United States) with idiosyncratic opportunities for implementing a variety of experimental designs and protocols covering both payoffs calibration patterns and probabilities calibration patterns that, together, have broad implications for plausibility of theories of decision under risk. 


\section{Dual Calibration Patterns and Dual Paradoxes}

For any given integer $m$, let $I_{m}$ denote the set of positive integers not larger than $m$, that is $I_{m}=\{1,2, \ldots, m\}$. Let $\left\{y_{k}, p_{k}\right\}_{k \in I_{m}}$ denote an $m$-outcome lottery, $L$ that pays $y_{k}$ with probability $p_{k}, k \in I_{m}$, where $\sum_{k=1}^{m} p_{k}=1$. We use the convention $y_{j+1} \geq y_{j}$, for $j \in I_{m-1}$. Whenever the smallest payoff is zero (i.e., $y_{1}=0$ ), we use the simpler notation $\left\{y_{m}, p_{m} ; \ldots ; y_{2}, p_{2}\right\}$.

In this paper we focus on a class of theories of decision under risk that includes all of the more prominent ones. The most familiar such theory is expected utility theory (EU) which represents the utility of a lottery, $L=\left\{y_{k}, p_{k}\right\}_{k \in I_{m}}$ with the functional

$$
(E U-1) \quad U(L)=\sum_{k=1}^{m} v\left(y_{k}\right) p_{k},
$$

where $v(\cdot)$ is a continuous positively-monotonic function that transforms payoffs. As is well known, EU represents risk aversion solely by concavity of the utility function $v(\cdot)$. The distinguishing property of functional (EU-1) is its linearity in probabilities, which follows from the Independence Axiom. ${ }^{3}$ With the terminal wealth model of EU, $v\left(y_{k}\right)=u\left(w+y_{k}\right), \forall k \in I_{m}$, where $w$ is the amount of initial wealth, whereas in case of the income model of EU the utility function $v(\cdot)$ is invariant to $w$.

In order to facilitate exposition of less familiar models, we will first rewrite (EU-1) in an alternative, logically equivalent form. Let $P_{k}, k \in I_{m}$ denote the (decumulative) probability that the lottery $L$ pays $y_{k}$ or more, that is $P_{k}=\sum_{i=k}^{m} p_{i}, k \in I_{m}$. Using this notation, (EU-1) can be rewritten as

$$
U(L)=v\left(y_{m}\right) P_{m}+\sum_{k=1}^{m-1} v\left(y_{k}\right)\left[P_{k}-P_{k+1}\right] .
$$

\footnotetext{
${ }^{3}$ This axiom was introduced by von Neumann and Morgenstern (1947). The statement of the axiom by Yaari (1987, pp.98) is: (IA) For all lotteries $A$ and $B$, if lottery $A$ is preferred to lottery $B$ then for all lotteries $C$, and all $\alpha \in[0,1]$, an $\alpha$-probability mixture of lotteries $A$ and $C$ is preferred to an $\alpha$-probability mixture of lotteries $B$ and $C$ : $\alpha A+(1-\alpha) C \succeq \alpha B+(1-\alpha) C$. That is getting lottery $A$ with probability $\alpha$ and lottery $C$ otherwise is preferred to the lottery that offers lottery $B$ with the same probability $\alpha$ and lottery $C$ otherwise.
} 
The class of theories of decision under risk that we consider have functionals that can be written in forms similar to (EU-2) but that relax the assumption of linearity in probabilities. The utility of a lottery, $L$ for this class of theories is given by

$$
U(L)=v\left(y_{m}\right) f\left(P_{m}\right)+\sum_{k=1}^{m-1} v\left(y_{k}\right)\left[f\left(P_{k}\right)-f\left(P_{k+1}\right)\right]
$$

where $f(\cdot)$ is a continuous, positively-monotonic function that transforms decumulative probabilities and, as above, $v(\cdot)$ is a continuous, positively-monotonic function that transforms payoffs. Of course, the EU functional is the special case of (NL-1) in which the probability transformation function is the identity mapping, $f(P)=P$.

Theories with functionals that are nonlinear in both payoffs and probabilities include rank dependent utility theory (Quiggin 1993) and cumulative prospect theory (Tversky and Kahneman 1992). We subsequently refer to this class of theories as NLPP theories.

In the case of dual theory of expected utility (DU), the functional is the special case of (NL1) that is linear in payoffs. The DU functional takes the simple form

$$
(D U-1) \quad U(L)=y_{m} f\left(P_{m}\right)+\sum_{k=1}^{m-1} y_{k}\left[f\left(P_{k}\right)-f\left(P_{k+1}\right)\right]
$$

that is dual to (EU-2). The distinguishing property of (DU-1), linearity in payoffs, follows from the Dual Independence Axiom (Yaari 1987). ${ }^{4}$ Recall that, for DU, risk aversion is equivalent to $f(\cdot)$ being convex (see Yaari 1987, p.107).

We begin with two examples that illustrate payoffs calibrations and probabilities calibrations. For ease of exposition, the examples build on EU and DU because of the simplicity that follows from linearity in either probabilities or payoffs. The propositions reported in sections 3 and 4, however, show that each of the dual patterns of risk aversion by itself has implausible implications for NLPP theories, such as rank dependent utility theory (Quiggin 1993) and cumulative prospect theory (Tversky and Kahneman 1992), that are characterized by functionals that are nonlinear in both payoffs and probabilities. As shown by Corollary 2.2 in section 4 , the

\footnotetext{
${ }^{4}$ The Dual Independence Axiom (Yaari 1987, pp.99) is: (DIA) For all lotteries $A$ and $B$, if lottery $A$ is preferred to lottery $B$, then for all lotteries $C$, and all $\alpha \in[0,1], \alpha$-payoff mixture of lotteries $A$ and $C$ is preferred to $\alpha$-payoff mixture of lotteries $B$ and $C$ : $\left(\alpha P_{A}^{-1}+(1-\alpha) P_{C}^{-1}\right)^{-1} \succeq\left(\alpha P_{B}^{-1}+(1-\alpha) P_{C}^{-1}\right)^{-1}$ where $(\cdot)^{-1}$ is a (generalized) inverse operator whereas $P_{A}$ and $P_{B}$ denote decumulative probability distribution functions of lotteries $A$ and $B$.
} 
probabilities calibration patterns described in section 2.2 also have implausible risk aversion implications for rank dependent models with endogenous reference amounts of payoff.

The first example, that illustrates payoffs calibration, builds on a pattern of small stakes risk aversion that appears in Rabin (2000). This pattern conforms to the dual theory of expected utility because of linearity of the DU functional in payoffs, as explained below. The second example, that illustrates probabilities calibration, builds on a pattern of risk aversion introduced in Sadiraj (2012). This second pattern conforms to expected utility theory because of linearity of the EU functional in probabilities, as explained below.

\subsection{Example 1: John Doe's Risk Preferences Conform to the Dual Theory of Expected Utility}

Suppose that John Doe, whose initial wealth is $\$ 125$, is observed to reject a 50/50 bet in which he could lose $\$ 100$ or gain $\$ 110$. Does decision theory make any predictions about what choice John would make at other wealth levels? Expected utility theory makes no prediction based on this one observation unless one adds special-case assumptions about risk attitudes such as constant or decreasing absolute risk aversion. In contrast, dual theory of expected utility (Yaari 1987) makes a general prediction about John's choices. According to the DU functional (as in (DU-1)), John's rejection of the 50/50 lose $\$ 100$ or gain $\$ 110$ bet at initial wealth level $\$ 125$ reveals $125 \geq U_{D U}($ bet $)=(125+110) f(0.5)+(125-100)(1-f(0.5))=125+210 f(0.5)-100$; thus $f(0.5) \leq 100 / 210$. From the last inequality it follows that $w \geq U_{D U}$ (bet) for all initial wealth $w \geq \$ 125$ because $w \geq U_{D U}($ bet $)=(w+110) f(0.5)+(w-100)(1-f(0.5))=w+210 f(0.5)-100$ is true if and only if $f(0.5) \leq 100 / 210$. Therefore, according to DU, John will (weakly) reject the bet at all wealth levels if he does so at wealth level $\$ 125$.

What are the implications for expected utility theory if one actually observes rejection of the bet or indifference for all initial wealth levels in some finite interval? Consider the pairs of lotteries in Table 1. The first row shows the alternative options discussed above, a choice between certain payoff $x=\$ 125$ in the option B column and a 50/50 bet, with outcomes $\$(x$ $100)$ or $\$(x+110)$ in the option A column. Now suppose that (as predicted by dual theory of expected utility) additional observations of John's decisions under risk show him choosing option B or indifference in all rows of Table 1. What are the implications of these observations for EU? EU implies that, if John weakly prefers the certain payoff to the lottery in all rows of 
Table 1, then he will also prefer a certain payoff of $\$ 3,000$ to a 50/50 bet with payoffs of $\$ 125$ or \$3.2 million (see Proposition 1 in section 3). What accounts for this implausible implication of EU?

According to EU, choice of option $B$ in the first row in Table 1 reveals that ${ }^{5}$ $v(125) \geq 0.5 v(235)+0.5 v(25)$, which together with the (weak) concavity of the continuously differentiable function, ${ }^{6} v(\cdot)$ imply that $110 v^{\prime}(235) \leq v(235)-v(125) \leq v(125)-v(25) \leq 100 v^{\prime}(25)$. Therefore $v^{\prime}(25+210) \leq(10 / 11) v^{\prime}(25)$. Choice of option B in the second row of Table 1 reveals that $v^{\prime}(25+2 \times 210) \leq(10 / 11) v^{\prime}(25+210)$, which together with the penultimate inequality imply $v^{\prime}(25+2 \times 210) \leq(10 / 11)^{2} v^{\prime}(25)$. Similarly, it can be verified that $v^{\prime}(25+210 t) \leq(10 / 11)^{t} v^{\prime}(25)$ follows from the weak preference for option B in all rows one to $t$ in Table 1. So, if option B is not rejected in all rows of Table 1, then the (weak) concave utility of payoffs explanation of these choices implies that

$$
v^{\prime}(24,175)=v^{\prime}(25+115 \times 210) \leq(10 / 11)^{115} v^{\prime}(25)<0.00002 \times v^{\prime}(25) .
$$

Such extreme diminishing marginal utility produces ridiculously low marginal utilities for large payoffs that leads to implausible large-stakes risk aversion such as the one stated at the end of the preceding paragraph.

\subsection{Example 2: Jane Doe's Risk Preferences Conform to Expected Utility Theory}

Now suppose that Jane Doe has been observed to reject a 50/50 bet that pays $\$ 30$ or $\$ 0$ in favor of a bet that pays $\$ 30$ with probability $0.45, \$ 10$ with probability 0.1 , or $\$ 0$ with probability 0.45 . Does decision theory make any predictions about what choice Jane would make when given options with higher or lower probability for the high payoff (of \$30) but with the same 0.1 probability of the middle outcome (of \$10)? DU makes no prediction based on this one observation unless one adds special-case assumptions about risk attitudes. In contrast, EU makes a general prediction about Jane's choices. Using the functional in statement (EU-1), one infers that Jane's rejection of the two-outcome bet reveals that $0.5 \times v(30) \leq 0.45 \times v(30)+0.1 \times v(10)$ if, without any loss of generality, the utility of outcome 0 is normalized to 0 . The last inequality simplifies to $0 \leq-0.05 v(30)+0.1 \times v(10)$. Adding $p \times v(30)$ to both sides of the immediately

\footnotetext{
${ }^{5}$ Recall that for the terminal wealth model $v\left(y_{k}\right)=u\left(w+y_{k}\right)$, whereas for the income model $v\left(y_{k}\right)=u\left(y_{k}\right)$.

${ }^{6}$ This illustrative example uses a differentiable utility function for simplicity. Concavity calibration does not require differentiability; see appendix A.1 or Rabin (2000).
} 
preceding inequality, one has $p \times v(30) \leq(p-0.05) \times v(30)+0.1 \times v(10)$. Hence, EU predicts that Jane will (weakly) reject the two-outcome lottery that pays $\$ 30$ with probability $p$ and $\$ 0$ otherwise, in favor of the three-outcome lottery that pays $\$ 30$ with probability $p-0.05, \$ 10$ with probability 0.1 and $\$ 0$ otherwise, for all $p \in\{0.05,0.1, \ldots, 0.95\}$, if she does so for $p=0.5$.

What are the implications for DU if one actually observes the choices that conform to EU, (weak) rejection of the two-outcome lottery in favor of the three-outcome lottery for all $p \in\{0.05,0.1, \ldots, 0.95\}$ ? Consider the pairs of lotteries in Table 2. Row 10 shows the first pair of options discussed above, a choice between a 50/50 bet that pays $\$ 30$ or $\$ 0$ in the option $\mathrm{A}$ column and a bet that pays $\$ 30$ with probability $9 / 20$, $\$ 10$ with probability $2 / 20$, or $\$ 0$ with probability $9 / 20$ in the option B column. Suppose that (as predicted by expected utility theory) observations of Jane's decisions under risk show her choosing option B or indifference in all rows of Table 2. Probabilities calibration (see section 4) shows that these observations have the implausible implication that Jane will also prefer a certain payoff of $\$ 3,000$ to a $50 / 50$ bet that pays $\$ 3$ million or $\$ 0$. What accounts for this implausible implication of DU?

Consider row 18 of Table 2. According to DU, the dual expected utilities of option $\mathrm{A}_{18}$ and option $\mathrm{B}_{18}$ are $U\left(A_{18}\right)=30 f(18 / 20)$ and $U\left(B_{18}\right)=30 f(17 / 20)+10[f(19 / 20)-f(17 / 20)]$. Subtraction of $10 f(18 / 20)$ from both $U\left(A_{18}\right)$ and $U\left(B_{18}\right)$, and rearrangement of terms shows that $U\left(B_{18}\right) \geq U\left(A_{18}\right)$ if and only if $[f(19 / 20)-f(18 / 20)] \geq 2[f(18 / 20)-f(17 / 20)]$. It follows from the last inequality and convexity of the continuously differentiable function, ${ }^{7} f(\cdot)$ that $f^{\prime}(19 / 20) \geq 2 f^{\prime}(17 / 20)$. Next, rejection of the two-outcome lottery in favor of the three outcome lottery in row 16 implies $f^{\prime}(17 / 20) \geq 2 f^{\prime}(15 / 20)$. Hence rejection of the two-outcome lottery in rows 16 and 18 reveals $f^{\prime}(19 / 20) \geq 2^{2} f^{\prime}(15 / 20)$. Similarly, rejection of the twooutcome lottery in all of the even-numbered rows of Table 2 reveals that

$$
f^{\prime}\left(\frac{19}{20}\right)=f^{\prime}\left(\frac{1+2 \times 9}{20}\right) \geq 2 f^{\prime}\left(\frac{1+2 \times 8}{20}\right) \geq \ldots \geq 2^{9} f^{\prime}\left(\frac{1}{20}\right)=512 f^{\prime}\left(\frac{1}{20}\right) .
$$

Such extreme increasing marginal probability transformation produces implausible risk aversion such as the one stated above.

\footnotetext{
${ }^{7}$ The proofs of Proposition 2 and its corollaries do not require either differentiability or convexity. To see that extreme implications follow for cases in which the probability transformation is not everywhere convex assume it has an inverted S-shape with an inflection point at $1 / 3$; in that case one still gets extreme risk aversion as revealed by: $f^{\prime}(19 / 20)=f^{\prime}((7+2 \times 6) / 20) \geq 2^{6} f^{\prime}(7 / 20)$.
} 


\subsection{Postulated Preferences for John and Jane Doe Are Paradoxical}

The postulated risk preferences for John and Jane Doe imply a double paradox: (a) John's pattern, that conforms to the dual theory of expected utility theory, has implausible risk aversion implications for expected utility theory; and (b) Jane's pattern, that conforms to expected utility theory, has implausible risk aversion implications for the dual theory of expected utility theory.

In sections 3 and 4 we offer a dual analysis of implications of patterns of risk aversion that (P.1) conform to the dual theory of expected utility or (P.2) conform to expected utility theory. The analysis shows that both patterns of type (P.1) and patterns of type (P.2) have implausible risk aversion implication for theories, such as rank dependent utility theory (Quiggin 1993) and cumulative prospect theory (Tversky and Kahneman 1992), with functionals that are nonlinear in both payoffs and probabilities.

The empirical relevance of these thought exercises with patterns of risk aversion rests on empirical validity of (weaker versions of) the patterns of risk aversion assumed in the above examples. We address this issue with the experiments reported in sections 5 and 6 . We next turn our attention to formal statements of calibration propositions and corollaries in sections 3 and 4.

\section{Payoffs Calibrations}

Calibration propositions for theories with nonlinear utility of money payoffs have been reported in several papers (cited above in the introduction). In order to provide a foundation for our payoffs calibration experiments, we report a calibration proposition for expected utility theory and a corollary that applies to rank-dependent theories on finite domains. The focus is on finite domains because of our intention to apply the theory to data from experiments. Design of experiments reported in section 5 is based on the calibration patterns discussed here.

\subsection{Calibration for Nonlinear Payoff Transformation Functions}

Consider payoff calibration patterns like those in Example 1 of section 2.1. For bounded intervals of income, Proposition 1 states a payoff calibration result for expected utility theory with weakly concave utility of money payoff function $u(\cdot){ }^{8}$

\footnotetext{
${ }^{8}$ See Rabin (2000) and Cox and Sadiraj (2006) for concavity calibrations on unbounded domains.
} 
The following standard notation is used: $\succeq$ indicates weak preference; $\succ$ indicates strong preference; $\{z, p ; y\}$ denotes a binary lottery that pays $z$ and $y$ with probabilities $p$ and $1-p$; $\lfloor x\rfloor$ is the largest integer smaller than $x$.

Let the domain of monetary prizes be a closed interval, $[m, M] \subset(0, \infty)$. For any given positive payoffs $\ell, g$ such that $0<\ell<g$ let $z_{*}=m+(\ell+g) \times(2-\ln 2 / \ln (\ell / g))$. Consider statements

P.1 $x \succeq\{x+g, 0.5 ; x-\ell\}$, for all integers $x \in[m, M]$

Q.1 $\{z+G, 0.5 ; m\} \succ z$,

for some $z \in\left(z_{*}, M\right)$ and sufficiently large $G>(z-m) g / \ell$.

Proposition 1. Let prizes $\ell, g$ such that $0<\ell<g$ and positive integer $m$ be given. Then

a. DU predicts that statement P. 1 is equivalent to $0 \succeq\{g, 0.5 ;-\ell\}$,

b. For all $z>z_{*}$ and all $G>(z-m) g / \ell$,

(i) for all $M>m$ both statements P.1 and Q.1 are true for DU with

$$
f(0.5) \in\left(\frac{z-m}{z-m+G}, \frac{\ell}{g+\ell}\right]
$$

(ii) there exists $M>m$ such that P.1 and Q.1 cannot both be true for EU.

Proof Parts (a) and (b.i) are straightforward; for part (b.ii) see appendix A.1.

Part (a) of this proposition says that any DU agent who rejects lottery $\{g, 0.5 ;-\ell\}$ will satisfy pattern P.1; the inverse is also true. Part (b) says, that for $G$ as large as one wants it to be, there are DU agents but no EU agents who satisfy both patterns P.1 and Q.1. The proof is constructive with respect to $M$. The following expression that relates $M$ to $G$ and $z$ (see the proof in appendix A.1, inequality (a.9)) will be useful in our numerical illustrations:

(*) $\quad G /(g+\ell)<N-K-1+r^{-N} A(r, K)$,

where $K=\lfloor(z-m) /(g+\ell)\rfloor, N=\lfloor(M-m) /(g+\ell)\rfloor, A(r, K)=r-r^{1+K}-r^{K}$ and $r=\ell / g$. We use statements $(*)$ and P.1 in Proposition 1 to construct the illustrative examples that are reported in Table 3 . 
Suppose that an agent weakly prefers the certain amount of income $x$ to the binary lottery $\{x+110,0.5 ; x-100\}$, for all integers $x \in[100, M]$, where values of $M$ are given in the "Rejection Intervals" column of Table 3. In that case all three expected utility (of terminal wealth, income, and initial wealth and income) models $^{9}$ predict that the agent prefers receiving the amount of income 3,000 for sure to a risky lottery $\{z+G, 0.5 ; 100\}$, where the values of $z+G\left(=G^{*}\right)$ are given in the second column of Table 3. For example, if $[m, M]=[100,50000]$ as in the last row of Table 3 , then $G^{*}=0.4 \times 10^{12}$; that is, expected utility theory implies that the agent will prefer 3,000 for sure to a $50 / 50$ lottery that pays 100 or $0.40 \times 10^{12}$. According to the entry in the third column and $M=30,000$ row of Table 3, expected utility theory predicts that if an agent prefers certain payoff in amount $x$ to lottery $\{x+90,0.5 ; x-50\}$, for all integers $x$ between 100 and 30,000, then such an agent will prefer 3,000 for sure to the 50/50 lottery with positive outcomes of 100 or $0.10 \times 10^{57}$.

\subsection{Calibration for Fixed Reference Payoff Models}

The generalization of Proposition 1 is straightforward for the NLPP class of theories that includes cumulative prospect theory with zero-income reference point (Tversky and Kahneman, 1992) and rank dependent utility theory (Quiggin, 1993). We use $R(p)$ to denote the following function $R(p)=(1-h(p)) \ell / h(p) g, p \in(0,1]$. One has:

Corollary 1.1. Suppose that the value function is (weakly) concave. For any positive prizes $\ell, g$ such that $R(0.5)<1$, NLPP theories predict that for all $z>z_{*}=m+(\ell+g)(2-\ln 2 / \ln (R(0.5))$ and any given large $G$ there exists $M$ such that $z, M$ and $G$ satisfy inequality (*) with $r=R(0.5)$ and statements P.1 and Q.1 cannot both be true.

Proof See appendix A.1.

Recall that for expected utility theory, with a functional that is linear in probabilities, Proposition 1 reveals implausible large-stakes risk aversion if $g>\ell$. In the corollary, this implication holds when $h(0.5) g>[1-h(0.5)] \ell$, which is equivalent to $r=R(0.5)<1$. Examples that illustrate the implications of Corollary 1.1 are similar to those in Table 3.

\footnotetext{
${ }^{9}$ See Cox and Sadiraj (2006) for discussion of these three expected utility models.
} 
A reference-dependent theory can incorporate variable reference amounts of money payoff. Wakker $(2005,2010)$ explains that variable reference payoffs can immunize prospect theory to payoffs calibration arguments based on the small-stakes risk aversion pattern (Rabin 2000). A straightforward extension of Wakker's arguments implies that the Markowitz (1952) model can be immunized to payoffs calibration by a suitable interpretation of its reference points.

Empirical validity of the P.1 pattern of small-stakes risk aversion is testable. Section 5 reports experiments on this question.

\section{Probabilities Calibrations}

We now consider varying probabilities, fixed payoffs patterns of risk aversion like those in Example 2 of section 2.2. We report a calibration proposition for the dual theory of expected utility and corollaries that apply to (NLPP) theories that have functionals that are nonlinear in both probabilities and payoffs. The design of experiments reported in section 6 is based on patterns of risk preferences discussed here.

\subsection{Calibration for Nonlinear Probability Transformation Functions}

Consider $2 n-1$ pairs of lotteries $A_{i}$ and $B_{i}, i \in I_{2 n-1}$. Lottery $A_{i}=\left\{y, p_{i}\right\}$ pays a positive amount of money $y$ with probability $p_{i}$ or the amount 0 with probability $1-p_{i}$. Denote $\delta=1 / 2 n$ and let $p_{i}=i / 2 n, i \in I_{2 n-1}$. Lottery $B_{i}=\left\{y, p_{i}-\delta ; x, 2 \delta\right\}$ pays the amount $y$ with probability $p_{i}-\delta$ or the amount $x, x \in(0, y)$ with probability $2 \delta$ or the amount 0 with probability $1-\left(p_{i}-\delta+2 \delta\right)$.

Suppose that an agent (weakly) prefers the three outcome lottery $B_{i}$ to the two outcome lottery $\mathrm{A}_{i}$, for all $i \in I_{2 n-k^{*}}$, where $k^{*}$ is a positive integer not larger than $n$. Note that by linearity in probabilities (see EU-1) any expected utility maximizer who prefers $x$ for sure to the 50/50 lottery that pays $y$ or 0 satisfies this supposition. Discussion following the statement of Proposition 2, however, shows that if the high outcome $y$ is larger than twice the intermediate outcome $x$ then this supposition implies implausible risk aversion for DU agents.

Let prizes $x, y$ such that $y>2 x>0$, and positive integer $k^{*}$ be given. For any integer $n$, $n \geq k^{*}$ consider the following statements

P.2 $\{y,(i-1) / 2 n ; x, 1 / n\} \succeq\{y, i / 2 n\}$, for all $i \in\left\{1,2, \ldots, 2 n-k^{*}\right\}$

Q.2 $\{z G, 0.5\} \succ\left\{z, 1-\left(k^{*}-1\right) / 2 n\right\}$, for some positive $z$ and large $G$. 
Proposition 2. Let prizes $x, y$ such that $y>2 x>0$, and positive integer $k^{*}$ be given. Then

a. For EU, statement P.2 is equivalent to $x \succeq\{y, 0.5\}$.

b. For any given sufficiently large $G$

(i) for all integers $n \geq k^{*}$ both statements P.2 and Q.2 are true for EU with $v(y)<2 v(x)$ and $2 v(1 / G)<v(1)$.

(ii) there exist integers $n \geq k^{*}$ such that statements P.2 and Q.2 cannot both be true for DU.

Proof Parts (a) and (b.i) are straightforward; for part (b.ii) see appendix A.2.

The proof is constructive with respect to $n$ and $G$. The following expression that relates $n$ to $G$ (see the proof in the appendix A.2, inequality (a.12)) will be useful in our numerical illustrations:

$$
\text { (**) } \quad G<T\left(y / x, n-k^{*}, n-1\right)
$$

where $T(t, m, n)=1+\sum_{j=0}^{m}(t-1)^{j+1} / \sum_{i=0}^{n}(t-1)^{-i}$. We use statements (**) and P.2 in Proposition 2 to construct the illustrative examples that are reported in Table 4 for the special case of $k^{*}=1$. In the table, $C$ denotes the ratio of the highest payoff to the second highest payoff in the three prize lottery, $C=y / x$. With $C=2.5$ and $n=20$, Proposition 2 tells us that for this P.2 pattern with DU predicts that the agent prefers 1,000 for sure to a lottery that pays 3.3 million or 0 with even odds, as reported in the second column and third row of Table 4. With $C=3.5$ and $n=50$, the prediction is preference for 1,000 for sure over a $50 / 50$ lottery that pays 0 or $0.78 \times 10^{23}$, as reported in the fourth column and fourth row of Table 4. Finally, with $C=5$ and $n=10$, the prediction is preference for 1,000 for sure to the 50/50 lottery that pays 0 or 1 billion.

\subsection{Calibration for Reference Dependent Models}

Proposition 2 is stated for the dual theory of expected utility that is characterized by a preference functional that is linear in payoffs and nonlinear in decumulative probabilities. The generalization is straightforward for the NLPP class of theories. First consider a NLPP model with fixed, zero-income reference point, as in Tversky and Kahneman (1992) and Quiggin 
(1993). For nonlinear transformation of payoff functions $v(\cdot)$ that are sub-additive on positive payoffs one has:

Corollary 2.1. For $v(y)>2 v(x)$, NLPP models with zero-income reference point predict that for any given sufficiently large $G$ there exist integers $n \geq k^{*}$ such that statements P.2 and Q.2 can't both be true.

Proof: see appendix A.2.

For NLPP models with zero-income reference point the relation between $n$ and $G$ is given by $G<T\left(v(y) / v(x), n-k^{*}, n-1\right)$. Implications of Corollary 2.1 for the special case in which $k^{*}=1$ are given in Table 4 for the (alternative) definition $C=v(y) / v(x)$. For example, if the value of the high payoff $v(y)$ is at least 3 times as large as the value of the intermediate (positive) payoff $v(x)$ then implications of calibration pattern P.2 are given by the $C=3$ column of Table 4 , and so on.

Probabilities calibration does not apply to the Markowitz (1952) model because its functional is linear in probabilities. In contrast, probabilities calibration applies to rank dependent models because of their nonlinear transformation of probabilities, whether or not the reference point is exogenous. The reason for this is straightforward: the calibration is constructed by varying the probabilities for which three or two payoffs are paid, not by varying the payoff amounts. Hence it makes no difference to this calibration whether the reference amount of payoff is or is not fixed at zero payoff. Here is a formal statement of the result that the calibration applies to rank dependent models with endogenous reference payoff. Let $\mu(\cdot)<0$ denote the value function for negative payoffs. For $v(\cdot)$ sub-additive on positive payoffs one has:

Corollary 2.2. Let the reference point be the intermediate payoff $x$ and $v(y-x)>-\mu(-x)$. Rank dependent models predict that for any given sufficiently large $\mathrm{G}$ there exist integers $n \geq k^{*}$ such that statements P.2 and Q.2 cannot both be true.

Proof See appendix A.2. 
It can be verified (see appendix A.2) that the relation between $n$ and $G$ in this case is given by $G<T\left(R+1, n-k^{*}, n-1\right)$, where $R=-v(y-x) / \mu(-x)$. Similar corollaries can be stated for cases in which the endogenous reference point is the highest payoff or the lowest payoff (or other convex combinations of the high and low payoffs) rather than the intermediate payoff. Empirical validity of the pattern P.2 is testable. Section 6 reports experiments on this question.

\section{Experiments with Varying Payoffs}

We ran three experiments with calibration patterns (P.1) for payoff transformation theories identified in Proposition 1 and Corollary 1.1 in Calcutta (India) and Magdeburg (Germany). We explain the common features and idiosyncratic lotteries used in these experiments after presenting a detailed discussion of one experiment to provide a representative example.

\subsection{Experimental Design: An Example}

Subjects in one experiment parameterization were asked to make six choices between a certain amount of money $x$ and a binary lottery $\{x+30,0.5 ; x-20\}$ for values of $x$ from the set $\{100$, $1 K, 2 K, 4 K, 5 K, 6 K\}$, where $K=1,000$. Subjects were asked to choose among option A (the risky lottery), option B (the certain amount of money), and option I (indifference). The choice tasks given to the subjects for this parameterization are presented in Table 5. Each row of Table 5 shows a certain amount of money and paired lottery in a choice task included in the experiment. The subjects were not presented with a fixed order of decision tasks, as in Table 5. Instead, each pair of a sure payoff and a risky lottery was shown on a separate (response form) page. Each subject picked up a set of response pages that were arranged in independently drawn random order. He or she could mark choices in any order desired.

\subsection{Experimental Design: Alternative Parameterizations and Protocols}

We conducted three experiments on empirical validity of the calibration pattern P.1 in Proposition 1. These experiments used the random decision selection payoff protocol in which one of each subject's several decisions is randomly selected for payoff at the end of the experiment. In the Calcutta $30 /-20$ experiment, binary lotteries $\{x+30,0.5 ; x-20\}$ and sure payoffs $x$ were from the set $\{100,1 K, 2 K, 4 K, 5 K, 6 K\}$, where $K=1,000$; payoffs were in rupees. In the Calcutta 90/-50 experiment, binary lotteries $\{x+90,0.5 ; x-50\}$ and sure payoffs 
$x$ were from the set $\{50,800,1.7 K, 2.7 K, 3.8 K, 5 K\}$, where $K=1,000$; payoffs were in rupees. Finally, in the Magdeburg $110 /-100$ experiment, binary lotteries $\{x+110,0.5 ; x-100\}$ and sure payoffs $x$ were from the set $\{3 K, 9 K, 50 K, 70 K, 90 K, 110 K\}$, where $K=1,000$; payoffs were in contingent euros.

An appendix available from the authors reports the subject instructions (in English), the response forms (or pages), and detailed information on the protocol used in all of the experiments. Before presenting data, we note economic significance of rupee payoffs in Calcutta experiments and the meaning of contingent euro payoffs in the Magdeburg experiment.

\subsection{Economic Significance of Payoffs}

Data collected in Calcutta at the time of the first experiment show that the 50 rupee amount at risk in the Calcutta 30/-20 experiment lotteries was the monetary equivalent of: (a) a full day's pay for the student subjects; (b) 15 (grocery store) servings of chicken; or (c) 14 bus tickets. Rupee payoffs in the Calcutta 90/-50 experiment were even more significant.

The Magdeburg 110/-100 experiment used contingent payoffs in amounts as high as $110 \mathrm{~K}$ euros. We could offer to pay such large amounts in contingent euros by using a casino payoff protocol explained in appendix A.3.

\subsection{Data Provide Support for the Concavity Calibration Pattern P.1}

Statement P.1 in Proposition 1 involves weak preference for (safe) option B over (risky) option A. Therefore, in all tests we aggregate choices of option B with (the very small number of) choices of option I (indifference) and denote the aggregated choice category as $\mathrm{B}^{\mathrm{I}}$. We report tests for incidence in the data of patterns of choices that, according to Proposition 1 and Corollary 1.1, imply implausible risk aversion in the large with expected utility theory and, for the experiments in Calcutta, with original cumulative prospect theory (with zero-income reference point) and with rank dependent utility theory.

We use error-rate analysis for statistical inferences on the proportion of subjects who made choices consistent with the calibration patterns. ${ }^{10}$ Choice probabilities are assumed to deviate from 1 or 0 by an error rate $\varepsilon$, as in Harless and Camerer (1994). Thus if $\mathrm{B}^{\mathrm{I}}$ is preferred to A then $\operatorname{Pr}\left(\right.$ choose $\left.\mathrm{B}^{\mathrm{I}}\right)=1-\varepsilon$ and if $\mathrm{B}^{\mathrm{I}}$ is not preferred to $\mathrm{A}$ then $\operatorname{Pr}\left(\right.$ choose $\left.\mathrm{B}^{\mathrm{I}}\right)=\varepsilon$, where $\varepsilon<0.5$. The

\footnotetext{
${ }^{10}$ We are grateful to Nathaniel Wilcox for generous advice about this approach to data analysis and for supplying SAS code. See Wilcox (2008) for discussion of econometric methods for analysis of data from binary discrete choice under risk.
} 
error rate model postulates that a subject with real preferences for $\mathrm{B}^{\mathrm{I}}$ (respectively $\mathrm{A}$ ) over $\mathrm{A}$ (respectively $\mathrm{B}^{\mathrm{I}}$ ) in all six rows could nevertheless be observed to have chosen $\mathrm{B}^{\mathrm{I}}$ in five (or fewer) out of six rows. That is, the model allows that a subject with real underlying preferences such as $\left[\mathrm{B}^{\mathrm{I}}, \mathrm{B}^{\mathrm{I}}, \mathrm{B}^{\mathrm{I}}, \mathrm{B}^{\mathrm{I}}, \mathrm{B}^{\mathrm{I}}, \mathrm{B}^{\mathrm{I}}\right]$ could, instead, choose a different pattern, say $\left[\mathrm{B}^{\mathrm{I}}, \mathrm{B}^{\mathrm{I}}, \mathrm{B}^{\mathrm{I}}, \mathrm{A}, \mathrm{B}^{\mathrm{I}}, \mathrm{B}^{\mathrm{I}}\right]$, an event with probability $(1-\varepsilon)^{5} \varepsilon$, where $\varepsilon$ is an error rate.

Models I, II, and III considered here are as follows. Model I includes only choices of all B (corresponding to $M=6,000$ in Proposition 1 for the Calcutta 30/-20 experiment, for example) as a calibration pattern and its mirror, all A's as the other pattern. Let the small stakes lotteries be $\{x+30,0.5 ; x-20\}$ for $x$ from 100 to 6,000 . According to Proposition 1 , the choice pattern "all $\mathrm{B}^{\mathrm{I} \text { ", }}$ implies that 1,000 for sure is preferred to the lottery that pays $0.13 \times 10^{23}$ or 100 with equal probabilities. Model II (which corresponds to Proposition 1 with $M=5,000$ for the Calcutta 30/20 experiment) contains the Model I pair of (calibration and other) patterns, and one additional calibration pattern with $\mathrm{A}$ as the last entry (for $x=6,000$ ) and its mirror image as an additional "other pattern." According to Proposition 1, the calibration patterns in Model II imply that getting 1,000 for sure is preferred to the $50 / 50$ lottery that pays $0.409 \times 10^{19}$ or 100 . Finally, Model III (which corresponds to Proposition 1 with $M=4,000$ for the Calcutta 30/-20 experiment) contains patterns with four sequential $\mathrm{B}^{\mathrm{I}}$ in the first four positions (for $x=100$, 1000, 2000, and 4000) as calibration patterns and their mirror images as other patterns. With these calibration patterns, Proposition 1 implies that getting 1,000 for sure is preferred to the lottery that pays $0.12 \times 10^{16}$ or 100 with equal probabilities.

The top row in Table 6 shows estimated proportions of subjects whose choices satisfy the calibration patterns for versions of Models I, II, and III using data from Calcutta 90/-50. The estimated proportion for Model I $(M=5,000)$ is 0.82 , with Wald 90 percent confidence interval $(0.70,0.94)$. The estimated proportions for all three models vary between 0.81 and 0.82 ; all are significant at one percent (indicated by superscripted double asterisks, **). The estimations for Calcutta 90/-50 imply that 81 to 82 percent of the subjects in this experiment made choices that conform to payoffs calibration patterns P.1 that are problematic for expected utility theory, rank dependent utility theory, and cumulative prospect theory with fixed reference point. The second row of Table 6 reports estimates for data from Calcutta 30/-20. The estimated proportions vary between 0.43 and 0.48 , and all are significant at one percent. Estimates in the third row for data from Magdeburg 110/-100 are 0.54; all are significant at one percent. 


\section{Experiments with Varying Probabilities}

We ran four probabilities calibration pattern P.2 experiments in Germany, India, and the United States. We explain the common design features and idiosyncratic lotteries in these experiments and present a more detailed discussion of one experiment to provide a representative example. We begin with the example.

\subsection{Experimental Design: An Example}

Subjects in one experiment parameterization were asked to make choices for each of the nine pairs of lotteries shown in Table 7. The fractions in the rows of the table are the probabilities of receiving the prizes in the two outcome (option A) and three outcome (option B) lotteries. Each row of Table 7 shows a pair of lotteries included in the experiment. The subjects were not presented with a fixed order of lottery pairs, as in Table 7. Instead, each lottery pair was shown on a separate (response form) page. Each subject picked up a set of response pages that were arranged in independently drawn random order. He or she could mark choices in any order desired. On each decision page, a subject was asked to choose among a two outcome lottery (option A in some row of Table 7), a three outcome lottery (option B in the same row of Table 7), and indifference (“option I").

\subsection{Experimental Design: Alternative Parameterizations and Protocols}

We conducted four experiments on empirical validity of the calibration pattern P.2 postulated in Proposition 2. One experiment parameterization uses pairs of two outcome and three outcome lotteries, $A_{j}=\left\{y, p_{j} ; 0\right\}$ and $B_{j}=\left\{y, p_{j}-0.1 ; x, 0.2 ; 0\right\}$, for $j \in\{1,2, \ldots, 9\}$, and $y=14, x=4$ as shown in Table 7. We also ran experiments with the parameterizations $\{y, x\}=\{40,10\}$ and $\{400,80\}$.

The experiments were conducted in Magdeburg (Germany), Atlanta (U.S.A.) and Calcutta (India) with payoffs, respectively, in euros, U.S. dollars, and Indian rupees. The experiments used the following parameters: in the Magdeburg 40/10 experiment, $y=40$ euros and $x=10$ euros; in the Atlanta 40/10 experiment, $y=40$ dollars and $x=10$ dollars; in the Atlanta 14/4 experiment, $y=14$ dollars and $x=4$ dollars; in the Calcutta 400/80 experiment, $y=400$ rupees and $x=80$ rupees. Economic significance of rupee payoffs is discussed in section 5.3. The two experiments in Atlanta each included two treatments that used different payoff protocols. One 
protocol is the conventional one in which a single decision by a subject is randomly selected for payoff at the end of the experiment; this is labeled POR, for "pay one randomly," in Table 8. The other payoff protocol is the one that is theoretically incentive compatible for dual theory of expected utility (Cox, Sadiraj, and Schmidt 2012). In this payoff protocol, all decisions are paid correlated at the end. In order to keep the level of incentives similar across payoff mechanisms, we used the version of the protocol in which the amounts of all payoffs are divided by the number of decisions; this is labeled PAC/9, for "pay all correlated/9," in Table 8. An appendix available from the authors reports subject instructions (in English), response forms (or pages), and detailed information on the protocol used in all of the experiments.

\subsection{Data Provide Support for Calibration Pattern P.2}

In testing for the presence of choices that satisfy the calibration pattern, we aggregate choices of option B with (the very small number of) choices of option I (indifference) because statement P.2 in Proposition 2 involves weak preference for B over A. Aggregated choices of B and I are reported as B $\mathrm{B}^{\mathrm{I}}$. Subjects' choice patterns are recorded as sequences of nine letters, ordered according to the probability of the high outcome. For example, the pattern $\left[A, B^{I}, B^{I}, A, B^{I}, B^{I}\right.$, $\left.\mathrm{B}^{\mathrm{I}}, \mathrm{B}^{\mathrm{I}}, \mathrm{A}\right]$ would indicate that a subject chose A (a two outcome lottery) when the probability of the high outcome was 1/10, 4/10 and 9/10 - indexed as $j=1,4$ and 9 - and chose B or I for all other values of the index $j$. For the experiment with parameterization shown in Table 7, this pattern would mean the subject chose option A on (randomly ordered) pages with the lottery pairs in rows 1,4 , and 9 in the table and chose option B or option I on all other pages.

We use error rate models to draw statistical conclusions from these data. The error rate model postulates that a subject with real preferences for $\mathrm{B}^{\mathrm{I}}$ (respectively A) over A (respectively $\mathrm{B}^{\mathrm{I}}$ ) in all nine lottery pairs could nevertheless be observed to have chosen the other option in some rows. For example, according to this model a subject with underlying preferences $\left[\mathrm{B}^{\mathrm{I}}, \mathrm{B}^{\mathrm{I}}\right.$, $\left.\mathrm{B}^{\mathrm{I}}, \mathrm{B}^{\mathrm{I}}, \mathrm{B}^{\mathrm{I}}, \mathrm{B}^{\mathrm{I}}, \mathrm{B}^{\mathrm{I}}, \mathrm{B}^{\mathrm{I}}, \mathrm{B}^{\mathrm{I}}\right]$ could, instead, be observed to choose a different pattern such as $\left[\mathrm{B}^{\mathrm{I}}, \mathrm{B}^{\mathrm{I}}\right.$, $\left.\mathrm{A}, \mathrm{B}^{\mathrm{I}}, \mathrm{A}, \mathrm{B}^{\mathrm{I}}, \mathrm{B}^{\mathrm{I}}, \mathrm{B}^{\mathrm{I}}, \mathrm{B}^{\mathrm{I}}\right]$, an event with probability $(1-\varepsilon)^{7} \varepsilon^{2}$.

Stochastic choice Model I contains only the choice pattern with a sequence of nine $\mathrm{B}^{\mathrm{I}}$ in the category "calibration pattern" and its dual ("mirror") image with a sequence of nine A in the "other pattern." According to Proposition 2, this calibration pattern implies that 1,000 for sure is preferred to the 50/50 lottery that pays 98,000 or 0 for the Atlanta 14/4 experiment, as reported 
in the third row in Table 8. For the Calcutta 400/80 experiment, Proposition 2 implies that 1,000 for sure is preferred to the 50/50 lottery that pays 1 million or 0 , as reported in the bottom row in Table 8.

Model I is overly conservative in its specification of calibration patterns because other data patterns can be calibrated to imply implausible risk aversion. Stochastic choice Model II includes two patterns in the category "calibration patterns": the pattern with choice of $\mathrm{B}^{\mathrm{I}}$ for index $j \in\{1,2, \ldots, 8\}$ and the all $\mathrm{B}^{\mathrm{I}}$ pattern (that is, $j \in\{1,2, \ldots, 9\}$ ). The mirror images of these two patterns comprise the "other patterns" for Model II. Application of Proposition 2 demonstrates that these two patterns of "no $A$ except for index $j=9$ " imply that 1,000 for sure is preferred to the 50/50 lottery that pays 81,000 or 0 , as reported for the Atlanta 40/10 and Magdeburg 40/10 listings in Table 8. We also consider Model III which includes the patterns "no $A$ except for indexes $j=8$ and/or 9" in the category of calibration patterns. The mirror images of these patterns comprise the other patterns for Model III. An implication of Proposition 2 for these calibration patterns in case of $n=5$ and $C=4$ is preference for 1,000 for sure to the $50 / 50$ lottery that pays 27,000 or 0, as shown in the Atlanta 40/10 and Magdeburg 40/10 listings in the table.

Table 8 reports results from maximum likelihood estimation of the proportion of subjects who exhibit the calibration patterns for Models I, II and III. The first row of Table 8 shows results for data from the Atlanta 14/4 experiment with POR payoff protocol. For Model I the estimated proportion of subjects who exhibited the calibration pattern is 0.74 . The Wald 90 percent confidence interval is $(0.55,0.93)$. The 0.74 estimate is significant at one percent (as indicated by double asterisks, **). The other columns in the first row of Table 8 report the estimated proportions of subjects whose choice patterns in the Atlanta 14/4, POR protocol treatment conform to calibration patterns of Models I, II, and III. These estimates vary between 0.74 and 0.88 , and they are all significant at one percent. Results look similar for the Atlanta 14/4, PAC/9 protocol data in the second row of Table 8 except that the proportions of subjects consistent with the calibration patterns are even higher; they vary from 0.81 to 0.93 .

Table 8 shows the estimated proportions of subjects whose choices are consistent with calibration patterns in experiments Atlanta 40/10, Magdeburg 40/10, and Calcutta 400/80. Depending on the model, the estimated proportion of subjects with data consistent with the calibration patterns in the Atlanta 40/10, POR protocol treatment varies from 0.56 to 0.59 , all significant at one percent. Results look somewhat different for the Atlanta 40/10, PAC/9 protocol 
data; here, the estimated proportions of subjects with choices consistent with the calibration patterns again are higher; they vary from 0.76 to 0.93 . The estimates for data from Magdeburg $40 / 10$ vary from 0.65 to 0.71 , all significant at one percent. Estimates with data from experiment Calcutta 400/80 lie between 0.72 and 0.73 ; all are significant at one percent.

\section{Is There a Plausible Theory for Decision under Risk?}

Prominent theories of decision under risk model individuals' preferences over lotteries with nonlinear transformation of money payoffs and/or nonlinear transformation of probabilities. Previous calibration literature, sparked by Rabin (2000), has focused on the implications of nonlinear transformation of payoffs. Sadiraj (2012) offers calibrations that focus on the implications of nonlinear transformation of probabilities. Theories with functionals that are nonlinear in both probabilities and payoffs are vulnerable to both types of calibration. If one allows for variable reference amounts of payoff, the probabilities calibration is problematic for these theories but the payoffs calibrations appearing elsewhere in the literature are not.

This paper develops a dual analysis of the calibration patterns. Taken together, the two calibration propositions provide a paradoxical insight into theories of decision under risk: a pattern of risk aversion that conforms to rational behavior for a theory with utility functional that is linear in probabilities (respectively, linear in payoffs) has implausible implications for a theory with functional that is linear in payoffs (respectively, linear in probabilities).

The internal consistency problems that follow from probabilities calibration are even more problematic than those from payoffs calibration. Whereas, the payoffs calibration critique applies across "small" and "large" payoff domains, the probabilities calibration critique also holds within a ("small" or "large") payoff domain.

First, note that the scale of the payoffs in pattern Q.1 depends on both the scale of payoffs in pattern P.1 and the length of the interval where P.1 holds. The scale of payoffs in pattern P.1 involves small-stakes risk aversion but the pattern Q.1 must characterize risk attitudes at largestakes to make the pair paradoxical. Alternatively, P.1 must involve small-stakes risk aversion to pair paradoxically with a given plausible Q.1 in the large. Therefore, varying-payoffs, fixedprobabilities calibrations question the ability of expected utility theory, rank dependent utility theory, and cumulative prospect theory with fixed reference point to rationalize plausible risk attitudes across different domains of risk: "small-stakes" and "large-stakes". 
In contrast, the scale of the payoffs in pattern P.2 is not required to be different from the scale of payoffs in pattern Q.2. ${ }^{11}$ Therefore, probabilities calibration questions the ability of dual theory of expected utility and rank dependent theories with exogenous (zero income) or endogenous reference point to rationalize plausible risk attitudes not only across different domains of payoffs (small versus large) but also within the same domain of payoffs.

Previous literature has not offered real-payoff, controlled experiment data on patterns of risk aversion that appear in calibration suppositions. This paper reports data from several experiments. As explained in section 6, the data provide support for empirical validity of risk aversion patterns underlying both of the dual calibrations.

Popular theories of decision under risk have been shown to be vulnerable to calibration critique. This suggests a central question: What properties would characterize a theory of riskavoiding preferences that would not be called into question by the critique? The answer follows from the dual calibration propositions and corollaries presented in this paper. A theory of risk preferences with a functional that is linear in probabilities would be immune to the probabilities calibration critique. A theory with variable reference payoffs would be immune to the payoffs calibration critique. The vintage Markowitz (1952, pp. 154-155) model has these two properties if one identifies the second inflection point with the windfall gain offered by the sure option in the payoff calibration pattern. ${ }^{12}$ Although a version of the Markowitz model does survive the critique, unlike all of the currently popular models, whether the former can survive other experimental tests is a question that needs to be addressed in subsequent research.

\footnotetext{
${ }^{11}$ For example, Proposition 2 and its corollaries tell us that the following two statements are inconsistent for DU and NLPP models. Statement P.2e: The three outcome lottery that pays 400 or 80 or 0 with probabilities $p-0.1,0.2$ and $1-(p-0.1+0.2)$ is preferred to the two outcome lottery that pays 400 or 0 with probabilities $p$ and $1-p$, for all $p$ in $\{0.1,0.2, \ldots, 0.8,0.9\}$. Statement Q.2e: The $50 / 50$ lottery that pays 330 or 0 is preferred to a sure payoff of 10 . These statements P.2e and Q.2e involve implausible combinations of same-stakes risk aversion. Furthermore, this same-stakes implausible risk aversion holds for all scales of payoffs because the P.2e and Q.2e statements are dimension-invariant; that is, the numbers $400,80,330$ and 10 could refer to numbers of cents, dollars or thousands of dollars or millions of dollars or any other payoff scale. The figures 330 and 1 in statement Q.2e apply for all utility, $v(\cdot)$ of prizes that satisfy $v(400) / v(80) \geq 3$.

${ }^{12}$ Unlike cumulative prospect theory with loss aversion, in a neighborhood of the origin the Markowitz model's transformation function for payoffs is convex on positive changes and concave on negative changes. How can one get risk aversion of type P.1 with this model? As an example think of the function being $x^{2}$ on the right of the origin up to the third inflection point and being $x^{3}$ on the left of the origin down to the first inflection point, where both the first and the third inflection points are further from the origin than the gains $(g)$ and the losses $(\ell)$ in pattern P.1.
} 


\section{Appendix}

\section{A.1. Proof of Proposition 1 and Corollary 1}

We state a general Proposition A.1 that applies to a theory with utility functional $U$ as in statement (NL-1). Proposition 1 and Corollary 1.1 follow directly from Proposition A.1.

General result $1 .^{13}$ Let a decision theory with "utility functional" $U$ as in statement (NL-1) be given. Referring to statement P.1, denote $a=\ell, b=g+\ell$ and let $N$ be the largest integer smaller than $(M-m) / b$. For a general probability, $p$ of the large outcome, $x+g$ statement P.1 for a sure outcome $x+a$ becomes

(a.i) $\quad x+a \succeq\{x+b, p ; x\}$, for all integers $x \in[m+a, M+a]$.

Suppose that ${ }^{14}$

(a.ii) $\quad b f(p)>a$,

where $f(\cdot)$ is the transformation function for decumulative probabilities as stated in the main text. Let $z_{*}=m+b(2-\ln 2 / \ln q)$, where $q=(1-f(p)) \ell / f(p) g$. Let $K$ be the largest integer smaller than $(z-m) / b$, for a given $z \in\left(z_{*}, M\right)$, and $J$ be the largest integer smaller than $N-K+1+A(q, K) q^{-N}$, where $A(q, K)=q-q^{1+K}-q^{K}$.

Proposition A.1. For all (weakly) concave function, $v(\cdot)$ and all positive numbers $a, b$ and $f(p)$ that satisfy (a.ii) one has:

1. For all $M>m$, for all $z \in\left(z_{*}, m+b N\right)$, statement (a.i) implies $z \succ\{z+(J-1) b, p ; m\}$;

2. For all positive $G$, for all $z>z_{*}$ there exists $N$ such that statement (a.i) implies that $z \succ\{z+G, p ; m\}$

Proof The proof of A.1.1 consists of two steps.

\footnotetext{
${ }^{13}$ This part of the proof is similar to Cox and Sadiraj (2006, pp.58).

${ }^{14}$ For theories that assume linearity in probabilities and $\mathrm{p}=0.5$, condition (a.ii) is simply $g>\ell$; it says that the expected value of risky lottery $\{b, 0.5 ; 0\}$ is larger than the sure amount of money $a$. For theories that assume nonlinearity in probabilities, condition (a.ii) says that the expected value of the risky lottery after applying the probability transformation, i.e. $\mathrm{EV}(\{b, f(p) ; 0\})$ is larger than the sure amount of money $a$.
} 
First, we show that statements (NL-1), (a.i) and (weak) concavity of $v(\cdot)$ imply that for all $x \in[m+a, m+N b+a]$

$$
v(x+j b)-v(x+(j-1) b) \leq q^{j}(v(x)-v(x-b)), \forall j \in \Psi_{x},
$$

where $\Psi_{x}$ is the set of all positive integers $j$ such that $x+(j-1) b-a$ belongs to the interval $[m, m+N b]$, that is $\Psi_{x}=\{j \in \mathrm{N} \mid x+(j-1) b-a \in[m, m+N b]\}$.

Then, we show that for any given $z \in\left(z_{*}, m+b N\right)$,

$$
m+K b \succeq\{m+(K+J) b, p ; m\} .
$$

That is getting $m+K b$ for sure is preferred to the binary lottery $\{m+(K+J) b, p ; m\}$, which completes the proof of part 1 since by construction of $K, m+(K+1) b>z \geq m+K b$ and therefore

$$
v(z) \geq v(m+K b) \geq f(p) v(m+(K+J) b)+(1-f(p)) v(m)>f(p) v(z+(J-1) b)+(1-f(p)) v(m) .
$$

It follows from statements (NL-1) and (a.i) that

$$
\text { (a.3) } \quad v(x+a) \geq(1-f(p)) v(x)+f(p) v(x+b), \forall x \in[m+a, m+N b+a] \cap \mathrm{N} .
$$

To derive (a.1) write $v(x+a)=f(p) v(x+a)+(1-f(p)) v(x+a)$ and rearrange terms in (a.3) to get

$$
\text { (a.4) }(1-f(p))[v(x+a)-v(x)] \geq f(p)[v(x+b)-v(x+a)], \forall x \in[m+a, m+N b+a] \cap \mathrm{N} \text {. }
$$

Note that from (weak) concavity of $v(\cdot),{ }^{15}(v(x)-v(x-g)) / g \geq(v(x+\ell)-v(x)) / \ell$, which together with statement (a.4) and using notations $q=(1-f(p)) \ell / f(p) g, a=\ell$ and $b=g+\ell$ imply

$$
\begin{aligned}
& v(x+b)-v(x)=v(x+b) \mp v(x+a)-v(x) \leq\left(\frac{1-f(p)}{f(p)}+1\right)(v(x+a)-v(x)) \leq \frac{1}{f(p)} \frac{\ell}{g}(v(x)-v(x-g)) \\
& =\frac{q}{1-f(p)}(v(x)-v(x-g))=q\left(1+\frac{f(p)}{1-f(p)}\right)(v(x)-v(x-g)) \leq q(v(x)-v(x-g)+v(x-g)-v(x-b)) \\
& =q(v(x)-v(x-b)) .
\end{aligned}
$$

Finally, statement (a.1) follows from applying $j$ times the preceding derived inequality, $v(x+b)-v(x) \leq q(v(x)-v(x-b))$.

\footnotetext{
${ }^{15}$ To see this write $x$ as a convex combination of $x+\ell$ and $x-g$ and apply the definition of concavity to get $v(x)=v([\ell(x-g)+g(x+\ell)] /(g+\ell)) \geq[\ell v(x-g)+g v(x+\ell)] /(g+\ell)$.
} 
To show statement (a.2), let $y=m+K b$ and verify that statement (a.1) implies

$$
v(y)-v(y-b K)=\sum_{k=0}^{K-1}[v(y-k b)-v(y-(k+1) b)] \geq \Delta v(y) \sum_{k=0}^{K-1}(1 / q)^{k},
$$

where $\Delta v(y)=v(y)-v(y-b)$. Next, it can be verified that $y \geq z-b>z_{*}-b$ implies that $K>1-\ln 2 / \ln q$. Hence $A(q, K)>0$ and therefore $J+K>N$, by construction of $J$. It follows from (weak) concavity of $v(\cdot)$ and statement (a.1) that

(a.6) $v(y+J b)-v(y)=\sum_{j=0}^{J-1}[v(y+(j+1) b)-v(y+j b)] \leq \Delta v(y)\left[q^{N-K+1}(J-N+K-1)+\sum_{j=0}^{N-K} q^{j+1}\right]$.

Statements (a.5) and (a.6) imply that a sufficient condition for (a.2) is

$$
(1-f(p)) \sum_{k=0}^{K-1}\left(1 / q^{k}\right)>f(p)\left[q^{N-K+1}(J-N+K-1)+\sum_{j=0}^{N-K} q^{j+1}\right] .
$$

Substitute $(1-q) \sum_{j=0}^{N-K} q^{j+1}=q\left(1-q^{N-K+1}\right),(1-q) \sum_{k=0}^{K-1} q^{-k}=q\left(q^{-K}-1\right)$ and $(1-f(p)) / f(p)=q g / \ell$ in (a.7) to get

$$
J<N-K+1+\left(\frac{g}{\ell}\left(q-q^{1+K}\right)-q^{K}+q^{1+N}\right) \frac{1}{(1-q) q^{N}} .
$$

To show that inequality (a.8) is indeed satisfied recall that $J \leq N-K+1+A(q, K) q^{-N}$ by construction, $q \in(0,1)$ by statement (a.ii) and verify that:

$$
A(q, K)=q-q^{1+K}-q^{K}<\left(\frac{g}{\ell}\left(q-q^{1+K}\right)-q^{K}+q^{N+1}\right) \frac{1}{(1-q)}
$$

Statement A.2.2 follows from statement A.1.1. Let $\mathrm{G}>0$ and $z>z_{*}$ be given. Then, as in part 1 , let $K$ be the largest integer smaller than $(z-m) / b$. It follows from $A(q, K)>0$ and $q \in(0,1)$ that there exists $N^{*}$ such that for all integers $N>N^{*}$, one has

$$
N-K-1+A(q, K) q^{-N}>G / b .
$$

If statement (a.i) is true for some $M$ such that $N=\lfloor(M-m) / b\rfloor$ satisfies (a.9) then one has: $z \succ\{z+(J-1) b, p ; m\} \succ\{z+G, p ; m\}$; the first inequality follows from part 1 (A.1.1) whereas the second one follows from the construction of $J$ and first-order-stochastic dominance. 
Proof of Proposition 1 (expected utility theory) Part a and Part b.i follow directly by linearity in payoffs of the DU functional (see DU-1) and $p=0.5$. Condition $G>(z-m) g / \ell$ implies that $0<(z-m) /(z-m+G)<\ell /(g+\ell)<1$. To show Part b.ii., note that statement (a.ii) is satisfied and apply part 2 of Proposition A.1 with $p=0.5, f(p)=p$ and $q=\ell / g$ to find $N$ such that statement P.1 for $M=m+N(g+\ell)$ implies $z \succ\{z+G, 0.5 ; m\}$, hence Q.1 is not true.

Proof of Corollary 1.1 (rank dependent utility theory) It is an application of Proposition A.1.2 with $p=0.5, f(p)=h(p)$ and $v(z)=v(z)$.

\section{A.2. Proof of Proposition 2 and its Corollaries}

General result 2. ${ }^{16}$ Let preferences over finite discrete lotteries, $L=\left\{x_{j}, p_{j}\right\}, j \in I_{m}$ be represented with utility functional as in statement (NL-1).

$$
U(L)=v\left(x_{m}\right) P_{m}+\sum_{j=1}^{m-1} v\left(x_{j}\right) \int_{P_{j+1}}^{P_{j}} d f
$$

where $P_{j}=\operatorname{Pr}\left(x: x \geq x_{j}\right)$ and $\int_{P_{j+1}}^{P_{j}} d f=f\left(P_{j}\right)-f\left(P_{j+1}\right)$. Without any loss of generality we use the normalization, $v(0)=0$.

Suppose that statement P.2 as stated above Proposition 2 holds; that is for some given $y \geq 2 x>0$, and positive integers $k^{*}$ and $n \geq k^{*}$, an agent prefers the following three outcome lottery to the binary one,

$$
\left\{y, p_{i}-\delta ; x, 2 \delta\right\} \succeq\left\{y, p_{i}\right\} \text {, for all } i \in\left\{1,2, \ldots, 2 n-k^{*}\right\},
$$

where $\delta=1 / 2 n$ and $\mathrm{p}_{i}=i / 2 n=i \delta$. Using notation $C \equiv v(y) / v(x)$ and function $T(\cdot)$ as defined in section 4.1 we first state and show a general Proposition A.2 for sub-additive value functions of prizes. Proposition 2 and its Corollaries follow straightforwardly from Proposition A.2.

\footnotetext{
${ }^{16}$ This part of the proof is similar to Sadiraj (2012).
} 
Proposition A.2. Suppose that statement P.2 is true. Then there exists integer $K$ such that $\left\{z, 1-\left(k^{*}-1\right) \delta\right\} \succ\{z K, 0.5\}$, for all $z>0$. The last statement is true for all integers $K$ that do not exceed $T\left(C, n-k^{*}, n-1\right)$.

Proof According to functional (NL-1), statement P.2 (i.e. equation (a.iv)) requires that

$$
v(x) \int_{(i-1) \delta}^{(i+1) \delta} d f+v(y) f((i-1) \delta) \geq v(y) f(i \delta), \quad \forall i \in\left\{1, \ldots, 2 n-k^{*}\right\},
$$

for some positive integer $k^{*}$ not larger than $n$. Adding and subtracting $v(x) f(i \delta)$ and rearranging terms in the last inequality we get

$$
\int_{i \delta}^{(i+1) \delta} d f \geq(C-1) \int_{(i-1) \delta}^{i \delta} d f, \forall i \in\left\{1, \ldots, 2 n-k^{*}\right\} .
$$

Write the last inequality for $i+k\left(=1, \ldots, 2 n-k^{*}\right\}$ and apply it $k$ times to get

$$
\int_{(i+k) \delta}^{(i+k+1) \delta} d f \geq(C-1) \int_{(i+k-1) \delta}^{(i+k) \delta} d f \geq \ldots \geq(C-1)^{k} \int_{i \delta}^{(i+1) \delta} d f
$$

which generalizes as

(a.10)

$$
\int_{j \delta}^{(j+1) \delta} d f \geq(C-1)^{i} \int_{(j-i) \delta}^{(j-i+1) \delta} d f, \quad \forall j \in\left\{i, \ldots, 2 n-k^{*}\right\} .
$$

To complete the proof it suffices to show that

$$
f(0.5) \leq \sum_{j=0}^{n-1}\left(\frac{1}{C-1}\right)^{j} \int_{0.5-\delta}^{0.5} d f \text { and } f\left(\left(2 n-k^{*}+1\right) \delta\right)-f(0.5) \geq \sum_{i=0}^{n-k^{*}}(C-1)^{i+1} \int_{0.5-\delta}^{0.5} d f
$$

because two inequalities in (a.11) imply that

$$
f\left(\left(2 n-k^{*}+1\right) \delta\right) \geq f(0.5)\left[1+\sum_{i=0}^{n-k^{*}}(C-1)^{i+1} / \sum_{j=0}^{n-1}(C-1)^{-j}\right] .
$$

Multiplying both sides of the last inequality by $v(z)$ and using the sub-additivity of $v(z)$ we get the needed result:

$$
v(z) f\left(\left(2 n-k^{*}+1\right) \delta\right) \geq f(0.5) v(z) T\left(C, n-k^{*}, n-1\right) \geq f(0.5) v(z K) .
$$

To show the first inequality of (a.11) verify that it follows from inequality (a.10) that 


$$
f(0.5)=\sum_{i=1}^{n} \int_{(i-1) \delta}^{i \delta} d f \leq \sum_{i=1}^{n}\left(\frac{1}{C-1}\right)^{n-i} \int_{(n-1) \delta}^{n \delta} d f=\sum_{j=0}^{n-1}\left(\frac{1}{C-1}\right)^{j} \int_{0.5-\delta}^{0.5} d f
$$

Similarly, to show the second inequality of (a.11) verify that

$$
f\left(\left(2 n-k^{*}+1\right) \delta\right)-f(0.5)=\sum_{j=n+1}^{2 n-k^{*}+1} \int_{(j-1) \delta}^{j \delta} d f \geq \sum_{j=n+1}^{2 n-k^{*}+1}(C-1)^{j-n} \int_{0.5-\delta}^{0.5} d f=\sum_{i=0}^{n-k^{*}}(C-1)^{i+1} \int_{0.5-\delta}^{0.5} d f
$$

Proof of Proposition 2 (dual theory of expected utility) Part (a) follows from the linearity in probabilities of the EU functional (see EU-1). For part (b.i) first note that $k^{*} \geq 1$ implies $\left(1-\left(k^{*}-1\right) \delta\right) v(z) \leq v(z)$; hence $v(z)<0.5 v(z G)$ is a sufficient condition for the inequality in statement Q.2 to be true. Letting $z=1 / G,{ }^{17}$ the last inequality is equivalently written as $v(1 / G)<0.5 v(1)$ which is satisfied for $G$ large enough because the right hand side is a positive finite number whereas the left hand side approaches 0 when $G$ is large enough. Next, any such EU agent, with $v(y)<2 v(x)$ clearly satisfies pattern P.2 (which follows from part a).

To show part (b.ii) first note that $y>2 x>0$ imply that $T\left(y / x, n-k^{*}, n-1\right)$ can be as large as one wants it to be for big enough $n$. So, for any given $G$ there exists $n \in N$ such that

$$
G<T\left(y / x, n-k^{*}, n-1\right) .
$$

Then apply Proposition A.2 with $v(z)=z$ to show that P.2 implies $\left\{z, 1-\left(k^{*}-1\right) \delta\right\} \succ\{z G, 0.5\}$, for all positive $z$. Therefore statement Q.2 is not true.

Proof of Corollary 2.1 (zero-income reference-dependent preferences) The proof is similar to the proof of Proposition 2.b.ii. There is only one difference: use $v(z)=v(z)$ instead of $v(z)=z$.

Proof of Corollary 2.2 (endogenous reference-dependent preferences) Let the endogenous reference point be the middle prize, $x$ and let $R$ denote the ratio between the value of the perceived gain and the absolute value of the perceived loss, that is $R=-v(y-x) / \mu(-x)>1$. Statement P.2 in this case implies

$$
\mu(-x) f^{-}(1-(i+1) \delta)+v\left((y-x) f^{+}((i-1) \delta) \geq \mu(-x) f^{-}(1-i \delta)+v(y-x) f^{+}(i \delta), \forall i \in I_{2 n-k^{*}},\right.
$$

\footnotetext{
${ }^{17}$ If one is interested in some particular positive $t$ different from 1 that we consider here, then take $z=t / G$ and verify that the sufficient condition becomes $v(t / G)<0.5 v(t)$, which is satisfied for $G$ large enough.
} 
which can be equivalently rewritten as

$$
\int_{i \delta}^{(i+1) \delta} d f^{+}=\int_{1-(i+1) \delta}^{1-i \delta} d f^{-} \geq \frac{v(y-x)}{-\mu(-x)} \int_{(i-1) \delta}^{i \delta} d f^{+}, \forall i \in I_{2 n-k^{*}},
$$

where the equality follows from $f^{+}(p)=1-f^{-}(1-p)$. Use notation $R$ and apply the last inequality $j-i$ times to get

$$
\int_{j \delta}^{(j+1) \delta} d f^{+} \geq R^{j-i} \int_{i \delta}^{(i+1) \delta} d f^{+}, \forall j \in\left\{i, \ldots, 2 n-k^{*}\right\},
$$

and then (as in the proof for the general result 2, Proposition A.2) verify that the following inequality is true

$$
f^{+}\left(1-\left(k^{*}-1\right) \delta\right) \geq f^{+}(0.5)\left[1+\sum_{j=0}^{n-k^{*}} R^{j+1} / \sum_{i=0}^{n-1} R^{-i}\right] .
$$

Finally, to complete the proof use the last inequality and sub-additivity of $v(\cdot)$, and choose $n$ such that $G<T\left(R+1, n-k^{*}, n-1\right)$; the existence of such $n$ follows from $R>1$.

\section{A.3 Contingent Euro Payoff Protocol}

The Magdeburg 110/-100 experiment included amounts $x$ that were as large as 110K euros. We could credibly offer to pay such large amounts in contingent euros by using the following protocol. The experiment included two parts. In part 1 subjects made their choices between the sure amounts and the lotteries in the MAX-Lab at the University of Magdeburg. They were told that whether their payoffs would be hypothetical or real depended on a condition which would be described later in part 2. After making their decisions the subjects were informed that real payoffs were conditional on winning gambles at the Magdeburg Casino. The payoff contingency was implemented in the following way. For each participant the experimenter placed $€ 90$ on the number 19 on one of the (four American) roulette wheels at the casino. The probability that this bet wins is $1 / 38$. If the bet wins, it pays 35 to 1 . If the first bet won, then the experimenter would bet all of the winnings on the number 23. If both the first and second bet won, then the payoff would be $€(35 \times 35 \times 90)=€ 110,250$, which would provide enough money to make it feasible to pay any of the amounts involved in the part 1 decision tasks for that subject. The real payoff contingency was made credible to the subjects by randomly selecting three of them to 
accompany the experimenter to the casino and subsequently report to the others whether the experimenter had correctly placed the bets and recorded the outcomes. 


\section{References}

Barberis, N., Huang, M., Thaler, R.H.: Individual Preferences, Monetary Gambles, and Stock Market Participation: A Case for Narrow Framing. American Economic Review 96, 1069-1090 (2006)

Bleichrodt, H., Eeckhoudt, L.: Saving Under Rank-Dependent Utility. Economic Theory 25, 505-511 (2005)

Boyle, G., Conniffe, D.: Compatibility of Expected Utility and $\mu / \sigma$ Approaches to Risk for a Class of non Location-

Scale Distributions. Economic Theory 35, 343-366 (2008)

Chade, H., de Serio, V.N.: Risk Aversion, Moral Hazard, and the Principal's Loss. Economic Theory 20, 637-644 (2002)

Chateauneuf, A., Cohen, M., Meilijson, I.: More Pessimism than Greediness: A Characterization of Monotone Risk Aversion in the Rank-Dependent Expected Utility Model. Economic Theory 25, 649-667 (2005)

Cox, J. C., Sadiraj, V.: Small- and Large-Stakes Risk Aversion: Implications of Concavity Calibration for Decision Theory. Games and Economic Behavior 56, 45-60 (2006)

Cox, J..C., Sadiraj, V., Schmidt, U.: Paradoxes and Mechanisms for Choice under Risk. Experimental Economics Center Working Paper 2012-08, Georgia State University (2012)

Eichner, T., Wagener, A.: More on Parametric Characterizations of Risk Aversion and Prudence. Economic Theory 21, 895-900 (2003)

Hansson, B.: Risk Aversion as a Problem of Conjoint Measurement. In: Gardenfors, P., Sahlin, N.E. (eds.) Decisions, Probability, and Utility, pp. 136-158. Cambridge University Press, New York (1988)

Harless, D., Camerer, C.F.: The Predictive Utility of Generalized Expected Utility Theories. Econometrica 62, 1251-1290 (1994)

Hu, T.-W.: Expected Utility Theory from Frequentist Perspective. Economic Theory. Online First, 25 June (2009)

Lajeri, F., Nielsen, L.J.: Parametric Characterizations of Risk Aversion and Prudence. Economic Theory 15, 469-

$476(2000)$

LiCalzi, M.: Upper and Lower Bounds for Expected Utility. Economic Theory 16, 489-502 (2000)

Maccheroni, F.: Yaari's Dual Theory Without the Completeness Axiom. Economic Theory 23, 701-714 (2004)

Markowitz, H.: The Utility of Wealth. Journal of Political Economy 60, 151-158 (1952)

Neilson, W. S.: Calibration Results for Rank-Dependent Expected Utility. Economics Bulletin 4, 1-5 (2001)

von Neumann, J., Morgenstern, O.: Theory of Games and Economic Behavior. Princeton, NJ. Princeton University Press, sec. ed. (1947)

Quiggin, J.: Generalized Expected Utility Theory. The Rank-Dependent Model. Boston: Kluwer Academic Publishers (1993)

Rabin, M.: Risk Aversion and Expected Utility Theory: A Calibration Theorem. Econometrica 68, 1281- 1292 (2000)

Rieger, M.O., Wang, M.: Cumulative Prospect Theory and the St. Petersburg Paradox. Economic Theory 28, 665679 (2006)

Rubinstein, A.: Dilemmas of an Economic Theorist. Econometrica 74, 865-883 (2006)

Ryan, M.J., Vaithianathan, R.: Medical Insurance with Rank-Dependent Utility. Economic Theory 22, 689-698 (2003)

Sadiraj, V.: Probabilistic Risk Attitudes and Local Risk Aversion: a Paradox. Experimental Economics Center Working Paper 2012-07, Georgia State University (2012)

Safra, Z., Segal, U.: Calibration Results for Non-Expected Utility Theories. Econometrica 76, 1143-1166 (2008)

Safra, Z., Segal,U.: Risk Aversion in the Small and in the Large: Calibration Results for Betweenness Functionals. Journal of Risk and Uncertainty 38, 27-37 (2009)

Tversky, A., Kahneman, D.: Advances in Prospect Theory: Cumulative Representation of Uncertainty. Journal of Risk and Uncertainty 5, 297-323 (1992)

Wakker, P.P.: Formalizing Reference Dependence and Initial Wealth in Rabin's Calibration Theorem. Econometric Institute, Erasmus University, Rotterdam (2005)

Wakker, P. P.: Prospect Theory: For Risk and Ambiguity. Cambridge University Press (2010)

Wilcox, N.: Stochastic Models for Binary Discrete Choice Under Risk: A Critical Primer and Econometric Comparison. In: Cox, J.C., Harrison, G.W. (eds.) pp.197-292. Risk Aversion in Experiments. Bingley, UK: Emerald, Research in Experimental Economics (2008)

Yaari, M. E.: The Dual Theory of Choice under Risk. Econometrica 55, $95-115$ (1987)

Zambrano, E.: Expected Utility Inequalities: Theory and Applications. Economic Theory 36, 147-158 (2008) 
Table 1. Example 1: Varying-Payoffs Calibration Pattern

\begin{tabular}{|c|c|c|}
\hline Row & Option A & Option B \\
& $\{x-100,0.5 ; x+110\}$ & 125 \\
\hline 1 & 25 or 235 & 335 \\
\hline 2 & 235 or 445 & 545 \\
\hline 3 & 445 or 655 & $\cdots$ \\
\hline$\cdots$ & $\ldots$ & $125+210(\mathrm{t}-1)$ \\
\hline$t$ & $25+210(\mathrm{t}-1)$ or $25+210 t$ & $\ldots$ \\
\hline$\cdots$ & $\ldots$ & 23,855 \\
\hline 114 & 23,755 or 23,965 & 24,065 \\
\hline 115 & 23,965 or 24,175 & $x$ \\
\hline
\end{tabular}


Table 2. Example 2: Varying-Probabilities Calibration Pattern

\begin{tabular}{|c|c|c|c|c|c|}
\hline \multirow[t]{2}{*}{ Row } & \multicolumn{2}{|c|}{$\begin{array}{l}\text { Option A } \\
\{30, p ; 0\}\end{array}$} & \multicolumn{3}{|c|}{$\begin{array}{c}\text { Option B } \\
\{30, p-1 / 20 ; 10,2 / 20 ; 0\}\end{array}$} \\
\hline & Payoff 30 & Payoff 0 & Payoff 30 & Payoff 10 & Payoff 0 \\
\hline 1 & $1 / 20$ & $19 / 20$ & $0 / 20$ & $2 / 20$ & $18 / 20$ \\
\hline 2 & $2 / 20$ & $18 / 20$ & $1 / 20$ & $2 / 20$ & $17 / 20$ \\
\hline$\cdots$ & $\cdots$ & $\cdots$ & $\cdots$ & $\cdots$ & $\cdots$ \\
\hline 10 & $10 / 20$ & $10 / 20$ & $9 / 20$ & $2 / 20$ & $9 / 20$ \\
\hline$\cdots$ & $\cdots$ & $\cdots$ & $\cdots$ & $\cdots$ & $\cdots$ \\
\hline$i$ & $i / 20$ & $(20-i) / 20$ & $(i-1) / 20)$ & $2 / 20$ & $(19-i) / 20$ \\
\hline$\cdots$ & $\cdots$ & $\cdots$ & $\cdots$ & $\cdots$ & $\cdots$ \\
\hline 18 & $18 / 20$ & $2 / 20$ & $17 / 20$ & $2 / 20$ & $1 / 20$ \\
\hline 19 & $19 / 20$ & $1 / 20$ & $18 / 20$ & $2 / 20$ & $0 / 20$ \\
\hline
\end{tabular}


Table 3. Calibrations for Varying-Payoffs Patterns: $3,000 \succ\left\{G^{*}, 0.5 ; 100\right\}$

\begin{tabular}{|c|c|c|c|}
\hline $\begin{array}{c}\text { Rejection Intervals } \\
{[\mathbf{1 0 0}, \boldsymbol{M}]}\end{array}$ & $\begin{array}{c}\text { Calibration for } \\
\boldsymbol{g = 1 1 0}, \boldsymbol{\ell}=\mathbf{1 0 0}\end{array}$ & $\begin{array}{c}\text { Calibration for } \\
\boldsymbol{g}=\mathbf{9 0}, \boldsymbol{\ell}=\mathbf{5 0}\end{array}$ & $\begin{array}{c}\text { Calibration for } \\
\boldsymbol{g}=\mathbf{3 0}, \boldsymbol{\ell}=\mathbf{2 0}\end{array}$ \\
\hline$\underline{M}$ & $G^{*}$ & $G^{*}$ & $G^{*}$ \\
\hline 6,000 & 6,690 & $0.22 \times 10^{13}$ & $0.13 \times 10^{23}$ \\
\hline 8,000 & 9,913 & $0.85 \times 10^{16}$ & $0.14 \times 10^{30}$ \\
\hline 10,000 & 15,298 & $0.31 \times 10^{20}$ & $0.16 \times 10^{37}$ \\
\hline 30,000 & $0.47 \times 10^{8}$ & $0.10 \times 10^{57}$ & $0.44 \times 10^{107}$ \\
\hline 50,000 & $0.40 \times 10^{12}$ & $0.32 \times 10^{93}$ & $0.12 \times 10^{178}$ \\
\hline
\end{tabular}


Table 4. Calibrations for Varying-Probabilities Patterns: $1000 \succ\{\bar{G}, 0.5\}$

\begin{tabular}{|c|c|c|c|c|c|}
\hline $\begin{array}{c}\text { Rejection } \\
\text { Intervals }\end{array}$ & \multicolumn{5}{|c|}{ Calibrated values of $\overline{\boldsymbol{G}}$ for different values of $\boldsymbol{n}$ and $\boldsymbol{C}$} \\
\hline$\underline{\boldsymbol{n}}$ & $\mathbf{C = 2 . 5}$ & $\mathbf{C = 3}$ & $\mathbf{C = 3 . 5}$ & $\mathbf{C = 4 . 0}$ & $\mathbf{C = 5 . 0}$ \\
\hline 5 & 8,593 & 33,000 & 98,000 & 244,000 & $1,025,000$ \\
\hline 10 & 58,665 & $1,025,000$ & $9,530,000$ & $0.59 \times 10^{8}$ & $0.10 \times 10^{10}$ \\
\hline 20 & $3,326,256$ & $0.10 \times 10^{10}$ & $0.90 \times 10^{11}$ & $0.34 \times 10^{13}$ & $0.10 \times 10^{16}$ \\
\hline 50 & $0.63 \times 10^{12}$ & $0.11 \times 10^{19}$ & $0.78 \times 10^{23}$ & $0.71 \times 10^{27}$ & $0.12 \times 10^{34}$ \\
\hline 100 & $0.40 \times 10^{21}$ & $0.12 \times 10^{34}$ & $0.62 \times 10^{43}$ & $0.51 \times 10^{51}$ & $0.16 \times 10^{64}$ \\
\hline 200 & $0.16 \times 10^{39}$ & $0.16 \times 10^{64}$ & $0.38 \times 10^{83}$ & $0.26 \times 10^{99}$ & $0.25 \times 10^{124}$ \\
\hline 500 & $0.11 \times 10^{92}$ & $0.32 \times 10^{154}$ & $0.93 \times 10^{202}$ & $0.36 \times 10^{242}$ & $0.10 \times 10^{305}$ \\
\hline
\end{tabular}


Table 5. Choice Alternatives in Varying-Payoffs Experiment Calcutta 30/-20

\begin{tabular}{|c|c|c|}
\hline Row & Option A & Option B \\
\hline 1 & 80 or 130 & 100 \\
\hline 2 & 980 or 1,030 & 1,000 \\
\hline 3 & 1,980 or 2,030 & 2,000 \\
\hline 4 & 3,980 or 4,030 & 4,000 \\
\hline 5 & 4,980 or 5,030 & 5,000 \\
\hline 6 & 5,980 or 6,030 & 6,000 \\
\hline
\end{tabular}


Table 6. Maximum Likelihood Estimates of the Proportion of Subjects Who Exhibit Patterns P.1 and Predictions (not Q.1) for Payoffs Calibration

\begin{tabular}{|c|c|c|c|c|}
\hline Experiment & $\begin{array}{l}\text { Nr. of } \\
\text { subjects }\end{array}$ & Model I & Model II & Model III \\
\hline \multirow[t]{2}{*}{$\begin{array}{c}\text { Calcutta 90/-50 } \\
\mathbf{m}=\mathbf{5 0}\end{array}$} & \multirow{2}{*}{40} & $\begin{array}{c}0.82 * * \\
(0.70,0.94)\end{array}$ & $\begin{array}{c}0.81^{* *} \\
(0.69,0.93)\end{array}$ & $\begin{array}{c}0.81 * * \\
(0.69,0.93)\end{array}$ \\
\hline & & $\mathrm{M}=5,000: 1000\rangle\left\{0.32 \times 10^{11}, 0.5 ; 50\right\}$ & $\mathrm{M}=4,000: 1,000\rangle\left\{0.53 \times 10^{9}, 0.5 ; 50\right\}$ & $\mathrm{M}=3,000: 1,000\rangle\left\{0.86 \times 10^{7}, 0.5 ; 50\right\}$ \\
\hline \multirow{2}{*}{$\begin{array}{l}\text { Calcutta 30/-20 } \\
\quad \mathbf{m}=\mathbf{1 0 0}\end{array}$} & \multirow{2}{*}{30} & $\begin{array}{c}0.43 * * \\
(0.25,0.62)\end{array}$ & $\begin{array}{c}0.48 * * \\
(0.30,0.66)\end{array}$ & $\begin{array}{c}0.48 * * \\
(0.30,0.67)\end{array}$ \\
\hline & & $\mathrm{M}=6,000: 1,000\rangle\left\{0.13 \times 10^{23}, 0.5 ; 100\right\}$ & $\mathrm{M}=5,000: 1,000\rangle\left\{0.40 \times 10^{19}, 0.5 ; 100\right\}$ & $\mathrm{M}=4,000: 1,000\rangle\left\{0.12 \times 10^{16}, 0.5 ; 100\right\}$ \\
\hline \multirow{2}{*}{$\begin{array}{c}\text { Magdeburg 110/-100 } \\
\mathrm{m}=\mathbf{3 0 0 0}\end{array}$} & \multirow{2}{*}{41} & $\begin{array}{c}0.54 * * \\
(0.39,0.68)\end{array}$ & $\begin{array}{c}0.54 * * \\
(0.39,0.68)\end{array}$ & $\begin{array}{c}0.54 * * \\
(0.36,0.71)\end{array}$ \\
\hline & & $\mathrm{M}=110,000: 5,000\rangle\left\{0.12 \times 10^{23}, 0.5 ; 3,000\right\}$ & $\mathrm{M}=90,000: 5,000\rangle\left\{0.14 \times 10^{19}, 0.5 ; 3,000\right\}$ & $\mathrm{M}=70,000: 5,000\rangle\left\{0.17 \times 10^{15}, 0.5 ; 3,000\right\}$ \\
\hline
\end{tabular}


Table 7. Choice Alternatives in Varying-Probabilities Experiment Atlanta 14/4

\begin{tabular}{|c|c|c|c|c|c|}
\hline Row & \multicolumn{2}{|c|}{ Option A } & \multicolumn{3}{c|}{ Option B } \\
\hline & Payoff 14 & Payoff 0 & Payoff 14 & Payoff 4 & Payoff 0 \\
\hline 1 & $1 / 10$ & $9 / 10$ & $0 / 10$ & $2 / 10$ & $8 / 10$ \\
\hline 2 & $2 / 10$ & $8 / 10$ & $1 / 10$ & $2 / 10$ & $7 / 10$ \\
\hline 3 & $3 / 10$ & $7 / 10$ & $2 / 10$ & $2 / 10$ & $6 / 10$ \\
\hline 4 & $4 / 10$ & $6 / 10$ & $3 / 10$ & $2 / 10$ & $5 / 10$ \\
\hline 5 & $5 / 10$ & $5 / 10$ & $4 / 10$ & $2 / 10$ & $4 / 10$ \\
\hline 6 & $6 / 10$ & $4 / 10$ & $5 / 10$ & $2 / 10$ & $3 / 10$ \\
\hline 7 & $7 / 10$ & $3 / 10$ & $6 / 10$ & $2 / 10$ & $2 / 10$ \\
\hline 8 & $8 / 10$ & $2 / 10$ & $7 / 10$ & $2 / 10$ & $1 / 10$ \\
\hline 9 & $9 / 10$ & $1 / 10$ & $8 / 10$ & $2 / 10$ & $0 / 10$ \\
\hline
\end{tabular}


Table 8. Maximum Likelihood Estimates of the Proportion of Subjects Who Exhibit Patterns P.2 and Predictions (not Q.2) for Probabilities Calibration

\begin{tabular}{|c|c|c|c|c|}
\hline Experiment & $\begin{array}{l}\text { Nr. Of } \\
\text { Subjects }\end{array}$ & Model I & Model II & Model III \\
\hline \multirow{3}{*}{$\begin{array}{c}\text { Atlanta } \\
14 / 4\end{array}$} & $\begin{array}{c}39 \\
\text { POR }\end{array}$ & $\begin{array}{c}0.74 * * \\
(0.55,0.93)\end{array}$ & $\begin{array}{c}0.82 * * \\
(0.68,0.96)\end{array}$ & $\begin{array}{c}0.88 * * \\
(0.77,0.99)\end{array}$ \\
\hline & $\begin{array}{c}34 \\
\text { PAC/9 }\end{array}$ & $\begin{array}{c}0.81 * * \\
(0.61,1.0)\end{array}$ & $\begin{array}{c}0.90 * * \\
(0.76,1.0)\end{array}$ & $\begin{array}{c}0.93 * * \\
(0.81,1.0)\end{array}$ \\
\hline & & $1000>\{98000,0.5 ; 0\}$ & $1000>\{39000,0.5 ; 0\}$ & $1000>\{15700,0.5 ; 0\}$ \\
\hline \multirow{3}{*}{$\begin{array}{c}\text { Atlanta } \\
40 / 10\end{array}$} & $\begin{array}{c}22 \\
\text { POR }\end{array}$ & $\begin{array}{c}0.56^{* *} \\
(0.37,0.75)\end{array}$ & $\begin{array}{c}0.59 * * \\
(0.39,0.78)\end{array}$ & $\begin{array}{c}0.59 * * \\
(0.40,0.79)\end{array}$ \\
\hline & $\begin{array}{c}35 \\
\text { PAC/9 }\end{array}$ & $\begin{array}{c}0.76 * * \\
(0.56,0.95)\end{array}$ & $\begin{array}{c}0.83 * * \\
(0.66,1.0)\end{array}$ & $\begin{array}{c}0.93 * * \\
(0.85,1.0)\end{array}$ \\
\hline & & $1000\rangle\{244000,0.5 ; 0\}$ & $1000\rangle\{81000,0.5 ; 0\}$ & $1000>\{27000,0.5 ; 0\}$ \\
\hline \multirow{2}{*}{$\begin{array}{l}\text { Magdeburg } \\
\mathbf{4 0 / 1 0}\end{array}$} & \multirow{2}{*}{$\begin{array}{c}31 \\
\text { POR }\end{array}$} & $\begin{array}{c}0.65^{* *} \\
(0.49,0.81)\end{array}$ & $\begin{array}{c}0.67^{* *} \\
(0.51,0.83)\end{array}$ & $\begin{array}{c}0.71 * * \\
(0.56,0.87)\end{array}$ \\
\hline & & $1000\rangle\{244000,0.5 ; 0\}$ & $1000>\{81000,0.5 ; 0\}$ & $1000>\{27000,0.5 ; 0\}$ \\
\hline \multirow{2}{*}{$\begin{array}{c}\text { Calcutta } \\
\mathbf{4 0 0 / 8 0}\end{array}$} & \multirow{2}{*}{$\begin{array}{c}40 \\
\text { POR }\end{array}$} & $\begin{array}{c}0.72^{* *} \\
(0.58,0.86)\end{array}$ & $\begin{array}{c}0.72 * * \\
(0.58,0.86)\end{array}$ & $\begin{array}{c}0.73^{* *} \\
(0.59,0.86)\end{array}$ \\
\hline & & $1000>\{1$ million, $0.5 ; 0\}$ & $1000\rangle\{256000,0.5 ; 0\}$ & $1000\rangle\{64000,0.5 ; 0\}$ \\
\hline
\end{tabular}

